\title{
Prostaglandin E2 regulates osteoclastogenesis through the induction of RANKL in middle ear cholesteatoma.
}

Ryusuke Imai ${ }^{1,2}$, Takashi Sato², Mika Teraoํ․ Hiroyuki Murota1, Hidenori Inohara ${ }^{2}$, Ichiro Katayama ${ }^{1}$ ${ }^{1}$ Dermatology Department of Integrated Medicine, Osaka University Graduate School of Medicine ${ }^{2}$ Department of Otorhinolaryngology-Head and Neck Surgery, Osaka University Graduate School of Medicine

\section{Introduction}

Middle ear cholesteatoma is structurally similar to epidermal cyst composed of both stratified squamous epithelium and surrounding fibrosis tissue. In spite of benign tumor, cholesteatoma destructs adjacent bones and causes various complications such as hearing loss, vertigo, facial nerve palsy and brain abscess. In inflammatory bone diseases such as periodontitis and rheumatoid arthritis, receptor activator of nuclear factor $\mathrm{KB}$ ligand (RANKL)-mediated osteocalstogenesis is well known to play an important role in bone destruction. Infiltrated leukocytes produce PGE2 which induce RANKL expression in osteoblasts, synovial fibroblasts and gingival fibroblasts. The induction of RANKL is regulated by PGE2-EP4 signaling. On the other hand mechanism of bone destruction by cholesteatoma has been explored for many years, it has not been cleared.

In this study, we investigated whether RANKL-mediated osteoclastogensis is in cholesteatoma. We found that PGE2-EP3/4 signaling was important in RANKL expression and osteoclastogensis.

Fig. Illustration and histopathology of cholesteatoma
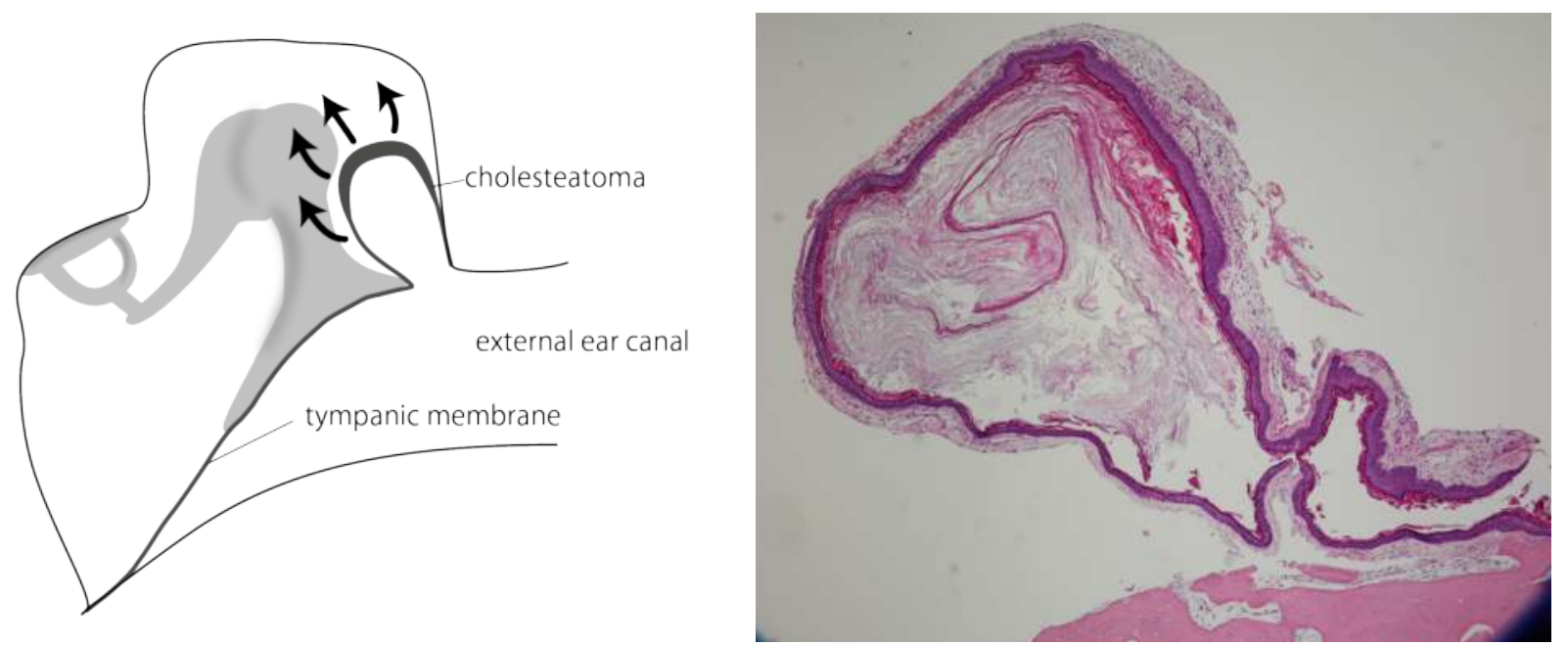

Result1. TRAP stained (red) osteoclasts increased in cholesteatoma.

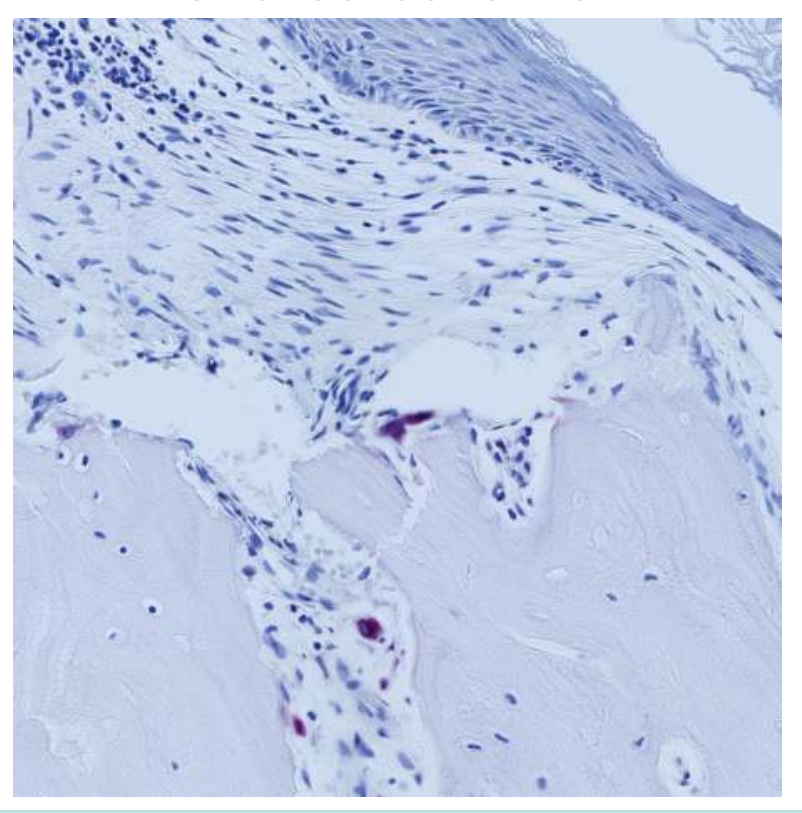

Result2. RANKL was expressed by fibroblasts in cholesteatoma.
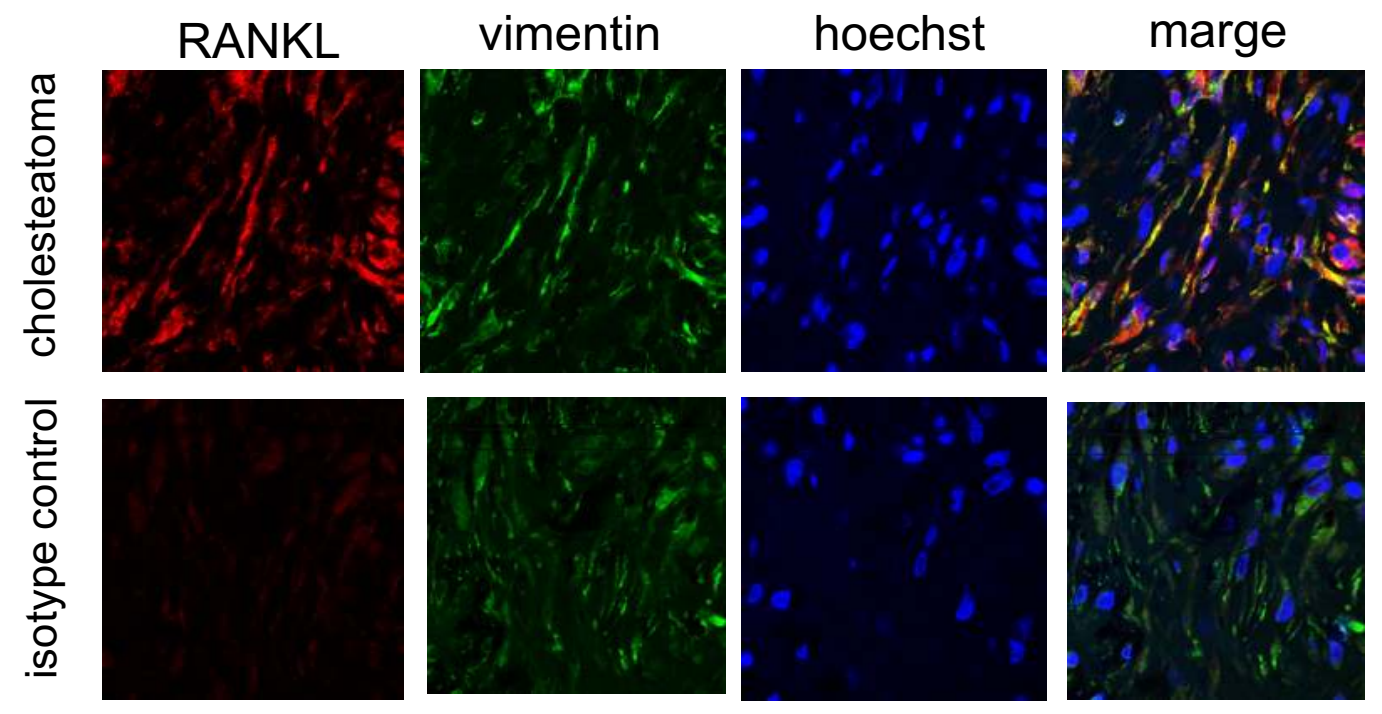

Result3. RANKL expression was enhanced by PGE2 stimulation, and this enhancement was suppressed by EP3 and EP4 antagonists in mouse fibroblasts from ear-pinna.

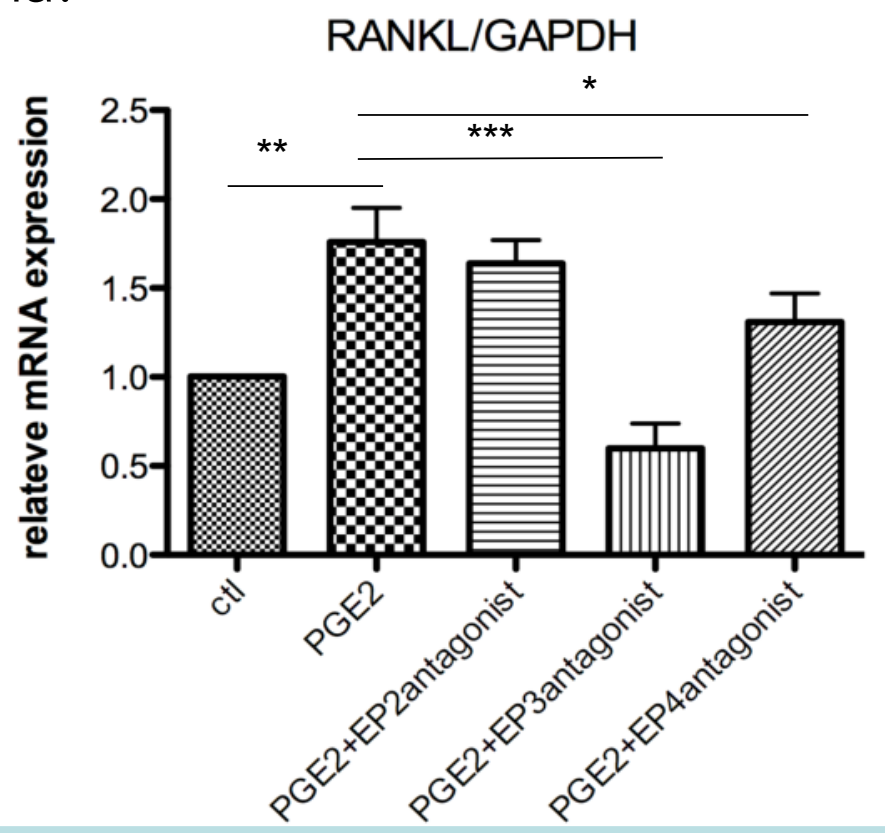

\section{Conclusion}

These data suggested that PGE2 regulated osteoclast differentiation through the induction of RANKL expression in fibroblasts of cholesteatoma.

Furthermore, these were implicated that cyclooxygenase-2 inhibitors or EP3 and EP4 antagonists have possibility of new therapeutic strategies for cholesteatoma-induced bone destruction. 